\title{
FACTORES ASOCIADOS A COMPLICACIONES DE LA COLANGIOPANCREATOGRAFÍA RETRÓGRADA ENDOSCÓPICA EN UN HOSPITAL DE ALTA COMPLEJIDAD
}

\author{
Angel Quispe-Mauricio 1,2,3,a,b, Wilmer Sierra-Cahuatat,a,c, David Callacondo, ${ }^{5,6, a}$, \\ Juan Torreblanca-Nava ${ }^{7, a, c}$
}

\begin{abstract}
RESUMEN
El tratamiento endoscópico de las enfermedades de la vía biliar es posible gracias a la colangiopancreatografía retrógrada endoscópica (CPRE); no obstante, no está exenta de complicaciones. Objetivos. Describir las características e indicaciones de la CPRE y determinar los factores asociados al desarrollo de complicaciones tras la realización de este procedimiento. Materiales y métodos. Se realizó un estudio observacional retrospectivo en el Departamento de Gastroenterología del Hospital Nacional Guillermo Almenara Irigoyen en Lima, Perú; desde marzo de 2002 a junio de 2005. Resultados. Se evaluaron 294 informes en 280 pacientes, la mediana de la edad fue 58 y 155 (52,7\%) fueron mujeres; cinco procedimientos se efectuaron en la Unidad de Cuidados Intensivos (UCI). La indicación más frecuente fue la coledocolitiasis en el 67,3\% de los casos, 205 (69,7\%) procedimientos fueron exitosos complicándose sólo 33 de ellos. Las complicaciones más frecuentes fueron la pancreatitis aguda y la hemorragia, en 16 y 13 pacientes, respectivamente. No se reportó casos de perforación o defunción. La canulación del conducto pancreático más de una vez fue un factor asociado (OR=2,01; IC95\%: 1,11 - 5,92; p=0,03). Conclusiones. El 11,2\% de los casos se complicaron, siendo la pancreatitis aguda y la hemorragia leve las complicaciones más frecuentes. Sólo la canulación al conducto pancreático en más de una oportunidad es un factor asociado para tener complicaciones.
\end{abstract}

Palabras clave: Pancreatocolangiografía retrógrada endoscópica; Complicaciones; Pancreatitis; Hemorragia; Canulación (fuente: DeCS BIREME).

\section{FACTORS ASSOCIATED TO COMPLICATIONS OF ENDOSCOPIC RETROGRADE CHOLANGIOPANCREATOGRAPHY IN A THIRD-LEVEL HOSPITAL}

\begin{abstract}
Endoscopic treatment of the bile duct diseases is possible thanks to the ERCP (endoscopic retrograde cholangio pancreatography), nevertheless, it is not free of complications. Objectives. To describe the characteristics and indications of the ERCP and determine the factors associated to the development of complications after performing the procedure. Materials and methods. An observational retrospective study was done in the Gastroenterology Department of the Hospital Guillermo Almenara Irigoyen in Lima, Peru, from March 2002 to June 2005. Results. 294 registers on 280 patients were evaluated, the median age was 58 and $155(52.7 \%)$ were women, five procedures we done in the intensive care unit (ICU). The most frequent indication was choledochus litiasis in $67.3 \%$ cases. 205 (69.7\%) procedures were successful, only 33 presented complications. The most frequent complications were acute pancreatitis and hemorrhage, in 16 and 13 patients respectively. There were no cases of perforation or death. Pancreatic duct cannulation more than once was an associated factor $(\mathrm{OR}=2.01 ; 95 \% \mathrm{Cl}: 1.11-5.92 ; \mathrm{p}=0.03)$. Conclusions. $11.2 \%$ of cases presented complications, being acute pancreatitis and mild hemorrhage the most frequent complications. Only pancreatic duct cannulation more than once is an associated factor for having complications.
\end{abstract}

Key words: Cholangiopancreatography, endoscopic retrograde; Complications; Pancreatitis; Hemorrhage; Catheterization (source: MeSH NLM).

\footnotetext{
1 Unité de Formation et de Recherche en Sciences de la Vie, Université Pierre et Marie Curie (Paris 6). París, Francia.

2 Faculté de Médicine Paris-Sud, Université Paris-Sud (Paris 11). París, Francia

3 Sociedad Científica de San Fernando, Universidad Nacional Mayor de San Marcos, Lima, Perú.

4 Servicio de Gastroenterología, Hospital Nacional Carlos Seguín Escobedo, EsSalud. Arequipa, Perú.

5 Departamento de Microbiología, Universidad Peruana Cayetano Heredia, Lima, Perú

6 Laboratorios de Investigación y Desarrollo, Facultad de Ciencias y Filosofía, Universidad Peruana Cayetano Heredia. Lima, Perú

7 Servicio de Gastroenterología, Hospital Nacional Guillermo Almenara Irigoyen, EsSalud. Lima, Perú.

Médico cirujano; ${ }^{\text {b } C a n d i d a t o ~ a ~ M a g i ́ s t e r ~ e n ~ C i e n c i a s ~ e n ~ B i o l o g i ́ a ~ C e l u l a r ~ y ~ M o l e c u l a r ; ~ ' ~ M e ́ d i c o ~ g a s t r o e n t e r o ́ l o g o . ~}$
}

Recibido: 04-02-10 Aprobado: 26-05-10 


\section{INTRODUCCIÓN}

La colangiopancreatografía retrógrada endoscópica (CPRE)es un procedimiento endoscópico gastrointestinal reportado por primera vez en 1968 por McCune et al., con una rápida aceptación como una técnica directa y segura para evaluar enfermedades biliares y pancreáticas. Con la introducción de la esfinterotomía endoscópica en 1974 por Kawai et al., la endoscopía terapéutica tuvo un rápido desarrollo, siendo en la actualidad una técnica de amplio uso (1).

Este procedimiento presenta una mayor morbilidad y mortalidad que la endoscopía alta, por lo que debe ser realizada por médicos especialistas entrenados, cuyo éxito va de la mano con un amplio entrenamiento adicional, así como experiencia en la realización de ésta para fines diagnósticos y terapéuticos ${ }^{(2,3)}$. La importancia de que el profesional esté preparado y capacitado para la realización de intervenciones terapéuticas al tiempo que realiza una CPRE diagnóstica, subyace en que muchos de los hallazgos pueden ser manejados con terapia endoscópica ${ }^{(4)}$.

La CPRE es una herramienta con la que cuentan hoy los centros hospitalarios de alto nivel de complejidad, donde se practica la cirugía de mínimo acceso, tanto intracavitaria como endoluminal ${ }^{(5)}$. Mediante este procedimiento se accede al sistema biliopancreático, para diagnosticar enfermedades primarias 0 complicaciones postquirúrgicas, además de permitir realizar en el momento, diversos tratamientos con un mínimo de riesgo ${ }^{(6)}$. Presenta menos complicaciones y disminuye la mortalidad postoperatoria en relación con la cirugía convencional, además de mejorar la calidad de vida de aquellos pacientes con enfermedades malignas reduciendo así los costos hospitalarios ${ }^{(5,7)}$.

Esta técnica es usualmente realizada bajo sedación intravenosa y analgesia, a menudo en una cita ambulatoria ${ }^{(3)}$. Los estudios de coagulación antes del procedimiento no están indicados rutinariamente, pero se deberían considerar en pacientes seleccionados, como aquellos con historia de coagulopatía o colestasis prolongada ${ }^{(3)}$. La profilaxis antibiótica se recomienda sólo en sospecha de obstrucción biliar, pseudoquiste pancreático conocido, historia previa de endocarditis o en pacientes portadores de válvulas cardiacas protésicas ${ }^{(8,9)}$.

En el diagnóstico de la enfermedad calculosa biliar, la colangiopancreatografía por resonancia magnética y la ultrasonografía endoscópica, han reemplazadoala CPRE diagnóstica pura ${ }^{(10)}$. Así también, existen alternativas a la CPRE terapéutica, como la cirugía laparoscópica e intervenciones radiológicas mínimamente invasivas como la colangiografía transparietohepática (CTPH). Sin embargo, la CPRE y sus indicaciones terapéuticas están bien definidas $y$, hasta la fecha, no ha podido ser superada por otras técnicas ${ }^{(11,12)}$, en tanto que su vigencia en el futuro, dependerá de la habilidad de los gastroenterólogos en demostrar sus ventajas en costos y resultados ${ }^{(13)}$

La pancreatitis es la complicación más común de la CPRE, con una incidencia reportada entre 1,8 y $7,2 \%$ en algunas series prospectivas ${ }^{(14-17)}$. No obstante, el reporte de incidencia puede variar ampliamente - hasta $40 \%$ dependiendo del criterio usado para el diagnóstico de pancreatitis, así como el tipo y duración de seguimiento al paciente ${ }^{(15)}$. Los criterios generalmente aceptados para el diagnóstico de pancreatitis post-CPRE, fueron expuestos en un Consenso en $1991^{(18)}$. Estos criterios incluyen la aparición de un nuevo dolor abdominal tipo pancreático, asociado con al menos un incremento de tres veces la amilasa sérica o lipasa, dentro de las 24 horas después de la CPRE. Asimismo, los síntomas de dolor necesitan ser lo suficientemente graves para requerir admisión a un hospital, o prolongar la estancia en pacientes que se encuentran hospitalizados ${ }^{(18)}$.

Otras complicaciones descritas incluyen hemorragia, sepsis de origen biliar, perforación de la vía biliar, hipoxemia arterial, arritmias e isquemia miocárdicas y una mortalidad de $0,12 \%$ a $0,9 \%{ }^{(2,16,19-22)}$. Asimismo, algunos problemas similares se han observado en otros procedimientos endoscópicos, especialmente en pacientes ancianos que padecen enfermedades cardíacas ${ }^{(23,24)}$. Se piensa que las complicaciones cardiopulmonares, ocurren al menos en el $60 \%$ de la morbilidad y el $50 \%$ de la mortalidad asociada con la endoscopía digestiva alta, y hasta un $50 \%$ de las muertes relacionadas con la CPRE (25).

Los factores de riesgo para el desarrollo de complicaciones post-CPRE, han sido clasificados en dos tipos, que incluyen a los factores propios del paciente (edad mayor de 60 años, sexo, cirrosis, diabetes mellitus, páncreas divisum, coagulopatía, colecistectomía y estar en una unidad de cuidados intensivos); y factores de riesgo del procedimiento (esfinterotomía, tamaño de la esfinterotomía, precorte, canulación del conducto pancreático, acinarización del páncreas, extracción de cálculos, diámetro del colédoco, tamaño del cálculo coledociano, anticoagulación y frecuencia de procedimientos realizadas por el endoscopista) ${ }^{(15,26)}$.

El Hospital Nacional Guillermo Almenara Irigoyen (HNGAI) cuenta con una alta y cada vez mayor demanda de pacientes asegurados de un importante 
sector poblacional de Lima y una parte de la población asignada del resto del país, siendo así un centro de referencia para este procedimiento por lo que el estudio de los factores asociados con complicaciones serviría como base para la formulación de intervenciones que permitan su reducción.

El objetivo del estudio fue describir las características e indicaciones de la CPRE y determinar los factores asociados con el desarrollo de complicaciones tras la realización de este procedimiento en una serie de procedimientos efectuados en pacientes atendidos en el HNGAI.

\section{MATERIALES Y MÉTODOS}

\section{TIPO DE ESTUDIO}

Se llevó a cabo una investigación observacional retrospectiva, en el Servicio de Gastroenterología del Hospital Nacional Guillermo Almenara Irigoyen (HNGAI) en Lima, Perú; durante el período de marzo de 2002 a junio de 2005, mediante la revisión de historias clínicas del total de pacientes sometidos a CPRE, el estudio fue aprobado por el Comité de Ética del HNGAI.

\section{POBLACIÓN DE ESTUDIO}

Se incluyeron los procedimientos realizados en personas de 20 años a más, procedentes de consulta ambulatoria u hospitalización, cuyas historias clínicas cuenten con datos completos para las variables a estudiar y consignen el respectivo informe de CPRE. No se evaluaron a aquellos casos donde no se logró acceso duodenal, existió falla del equipo endoscópico o radiológico, que por consecuencia de complicaciones derivadas de la endoscopía o sedación fueron suspendidas.

\section{DEFINICIÓN DE TÉRMINOS}

La definición de CPRE y sus complicaciones fueron tomadas de Loperfido ${ }^{(19)}$. El éxito del procedimiento (CPRE) fue definido como aquel que logró el objetivo de su indicación (aquellas en las que se logró canular correctamente la papila con criterio principalmente terapéutico y mínimamente diagnóstico).

En cuanto a las complicaciones se consideró lo siguientes criterios:

- Pancreatitis (días de hospitalización). Leve: 2-3; moderada: 4-10; grave: >10 días.
- Hemorragia (ejecución de transfusión sanguínea). Leve: ninguna; moderada: <4; grave: $>5$.

- Perforación: Leve: posible fuga de líquido o contraste o tratado con fluidos o succión por < 3 días; moderada: cualquier perforación detectable, tratada medicamente por 4-10 días; grave: tratamiento médico $>10$ días o necesidad de intervención quirúrgica o percutánea.

- Colangitis (según temperatura y grado de sepsis). Leve: $38^{\circ} \mathrm{C}$ por 1 a 2 días; moderada: >3 días; grave: shock séptico o requerimiento de cirugía ${ }^{(27)}$.

\section{RECOLECCIÓN DE DATOS}

Se diseñó un cuestionario que incluía las variables a estudiar, el cual fue evaluado y aprobado por juicio de expertos; conformado por ocho profesionales de la salud; evaluándose su concordancia por medio de procedimientos que implicaban el uso de la distribución binomial. Se tomaron los días hábiles de la semana para la recolección de datos en el Departamento de Registro y Archivos del HNGAI.

\section{ANÁLISIS ESTADÍSTICO}

A nivel descriptivo se usaron distribución de frecuencias para variables categóricas, en el caso de variables numéricas se usó medidas de tendencia central y de dispersión. A pesar de no contar con un muestreo probabilístico, se realizó un análisis bivariado, optándose por el test exacto de Fisher para evaluar asociación entre dos variables categóricas y el reporte del odds ratio con sus respectivos intervalos de confianza al $95 \%$.

Considerando la complicación como una variable dicotómica, se propuso realizar un análisis multivariado por medio de una regresión logística binaria con las variables que mostrasen asociación significativa a nivel bivariado. Se usó el paquete estadístico Statistical Packcage for Social Sciences (SPSS Inc. Chicago, Illinois) versión 15.0 para Windows.

\section{RESULTADOS}

De un total de 540 CPRE realizadas en el período de marzo de 2002 a junio de 2005, 294 informes fueron seleccionados por cumplir los criterios de elegibilidad del estudio. Los procedimientos evaluados fueron realizados en 280 pacientes, debido a que se indicó la repetición del procedimiento en 14 de ellos.

La mediana y rango intercuartílico de la edad fue 58 y 27 , respectivamente con un valor mínimo de 21 y un máximo 
Tabla 1. Indicaciones de CPRE en los procedimientos estudiados.

\begin{tabular}{lcr}
\hline Tipo de indicación & Frecuencia & $\%$ \\
\hline Sospecha de colédoco litiasis & 198 & 67,4 \\
NM periampular & 20 & 6,8 \\
Ictericia obstructiva & 17 & 5,8 \\
\hline Colangitis & 13 & 4,4 \\
Pancreatitis aguda biliar & 9 & 3,1 \\
\hline Quiste hidatídico & 8 & 2,7 \\
Colestasis & 7 & 2,4 \\
\hline Colocación y recambio de stent & 6 & 2,0 \\
Pancreatitis crónica & 5 & 1,7 \\
Pancreatitis de etiología por determinar & 3 & 1,0 \\
Otras & 8 & 2,7 \\
Total & 294 & 100,0 \\
\hline
\end{tabular}

de 91 años. Se observó una mayor frecuencia de mujeres que de hombres (52,7\% y $47,3 \%$ respectivamente), 289 procedimientos se realizaron en pacientes estables y el resto en $\mathrm{UCl}$. Con relación a los resultados sobre el éxito del procedimiento (CPRE), del total de CPRE realizadas, la mayoría $(69,7 \%)$ fueron exitosas.

En la Tabla 1 se presentan las indicaciones de CPRE, observándose que la indicación más frecuente fue la sospecha de coledocolitiasis, seguida de la evaluación de la vía biliar por neoplasia maligna periampular.

Dentro de los factores asociados a complicaciones propios del procedimiento tal como se reporta en la Tabla 2 , se observó que cuando se realizó la canulación del pancreático una o más veces, se obtuvo mayor frecuencia de complicaciones, siendo de $18,46 \%$, en comparación al $9,17 \%$ de complicaciones cuando no se canuló, produciéndose una diferencia significativa $(p=0,03)$; los otros factores presentados en la tabla no contaron con la significancia estadística suficiente para ser catalogados como asociados con la variable de interés. En aquellos pacientes que se efectuó esfinterotomía, se evaluó si el ser una esfinterotomía grande o pequeña se asociaría a complicación, el análisis estadístico mostró que no existe asociación estadísticamente significativa, similar a lo encontrado con el tamaño del cálculo en aquellos pacientes que se les extrajo cálculos.

Las complicaciones derivadas de CPRE se presentaron en el $11,2 \%$ del total de los procedimientos, como se muestra en la Tabla 3. La complicación más frecuente fue la pancreatitis aguda que ocurrió en 16 pacientes $(5,4 \%)$. La pancreatitis aguda fue catalogada como leve en 11 casos $(69 \%)$ y moderada en 5 casos $(31 \%)$; no hubo casos catalogados como graves. La hemorragia ocurrió en 13 pacientes $(4,4 \%)$, y fue catalogada como leve en el total de los casos; la colangitis y canastilla impactada ocurrieron en $2(0,7 \%)$ pacientes con la misma frecuencia. No se observó casos graves ni de mortalidad.

Tabla 2. Evaluación de la asociación entre factores propios del procedimiento y complicaciones en la Colangiopancreatografía Retrógrada Endoscópica en la población estudiada.

\begin{tabular}{|c|c|c|c|c|c|c|c|}
\hline Factores & $\begin{array}{c}\text { Total } \\
(n=294)\end{array}$ & $\begin{array}{c}\text { Complicaciones } \\
\qquad(n=33)\end{array}$ & $\begin{array}{l}\text { Proporción del factor } \\
\text { en procedimiento con } \\
\text { complicaciones (\%) }\end{array}$ & $\begin{array}{l}\text { Proporción del factor } \\
\text { en procedimiento sin } \\
\text { complicaciones }(\%)\end{array}$ & OR & IC al $95 \%$ & p \\
\hline Con esfinterotomía & 181 & 17 & \multirow{2}{*}{51,5} & \multirow{2}{*}{62,8} & \multirow{2}{*}{0,62} & \multirow{2}{*}{$0,28-1,39$} & \multirow{2}{*}{$0,21^{*}$} \\
\hline Sin esfinterotomía & 113 & 16 & & & & & \\
\hline Precorte & 11 & 0 & \multirow{2}{*}{0,0} & \multirow{2}{*}{3,7} & \multirow{2}{*}{0,00} & \multirow{2}{*}{$0-3,64^{£}$} & \multirow{2}{*}{$0,61^{*}$} \\
\hline No precorte & 283 & 33 & & & & & \\
\hline Canulación del c. pancreático >1 & 65 & 13 & \multirow{2}{*}{39,4} & \multirow{2}{*}{19,9} & \multirow{2}{*}{2,61} & \multirow{2}{*}{$1,11-5,92$} & \multirow{2}{*}{$0,02^{*}$} \\
\hline No canulación & 229 & 20 & & & & & \\
\hline Anticuagulado & 1 & 1 & \multirow{2}{*}{3,0} & \multirow{2}{*}{0,0} & \multirow{2}{*}{-} & \multirow{2}{*}{-} & \multirow{2}{*}{$0,11^{*}$} \\
\hline No anticuagulado & 293 & 32 & & & & & \\
\hline Extracción de cálculos >1 & 69 & 4 & \multirow{2}{*}{12,1} & \multirow{2}{*}{24,9} & \multirow{2}{*}{0,41} & \multirow{2}{*}{$0,10-1,25$} & \multirow{2}{*}{$0,12^{*}$} \\
\hline No Extracción de cálculos & 225 & 29 & & & & & \\
\hline Diámetro del colédoco $>10 \mathrm{~mm}$ & 118 & 15 & \multirow{2}{*}{45,5} & \multirow{2}{*}{40,4} & \multirow{2}{*}{1,23} & \multirow{2}{*}{$0,54-2,71$} & \multirow{2}{*}{$0,57^{*}$} \\
\hline Diámetro del colédoco <=10mm & 170 & 18 & & & & & \\
\hline
\end{tabular}

* Test exacto de Fisher,

${ }^{£}$ No puede darse el valor exacto del intervalo debido a no existir complicaciones en precorte. 
Tabla 3. Tipo de complicación según grado de severidad y frecuencia de complicaciones respecto al total de procedimientos CPRE efectuados.

\begin{tabular}{lcccccc}
\hline \multicolumn{1}{c}{ Tipo de complicación } & Leve & Moderada & Grave & N & \%* $^{*}$ & \%** $^{* *}$ \\
\hline Pancreatitis & 11 & 5 & - & 16 & 48,5 & $\mathbf{5 , 4}$ \\
Hemorragia & 13 & - & - & 13 & 39,4 & $\mathbf{4 , 4}$ \\
Colangitis & 1 & 1 & - & 2 & 6,1 & $\mathbf{0 , 7}$ \\
Canastilla Impactada & 2 & - & - & 2 & 6,1 & $\mathbf{0 , 7}$ \\
Perforación & - & - & - & - & - & - \\
Total & & & & $\mathbf{3 3}$ & $\mathbf{1 0 0 , 0 0}$ & $\mathbf{1 1 , 2}$ \\
\hline
\end{tabular}

* Con respecto al total de procedimientos complicados.

** Con respecto a la totalidad de procedimientos.

Cuando se evaluó la asociación entre la frecuencia de complicaciones según el éxito de la CPRE, no se encontró asociación entre estas variables $(O R=1,17$ y un $p=0,68)$. Del total de procedimientos sin éxito, 70 pacientes no presentaron complicaciones y sólo 11 de ellos las tuvieron. Mientras que del total de pacientes con éxito, 22 pacientes presentaron complicaciones y en tanto que 103 no las tuvieron.

Los factores propios del paciente que potencialmente se asocian a complicaciones post-CPRE son presentados en la Tabla 4, donde incluimos además, páncreas divisum en siete pacientes, coagulopatía en un paciente y cirrosis de dos pacientes. Ninguno de estos factores se asoció significativamente, asimismo, es relevante mencionar que no hubieron casos de defunción tras el procedimiento.

\section{DISCUSIÓN}

Desde la introducción de la esfinterotomía endoscópica y el desarrollo de stents biliares, la CPRE se ha convertido en un procedimiento de gran utilidad diagnóstica y terapéutica para el manejo de una variedad de enfermedades pancreáticas y biliares ${ }^{(28)}$.

La CPRE terapéutica con papiloesfinterotomía, para la extracción de cálculos de la vía biliar, colocación y recambio de stents biliares y pancreáticos es el procedimiento más difícil de la endoscopía digestiva. El éxito depende del entrenamiento, habilidad y la experiencia del endoscopista, y la tasa de éxito varía entre 90 a 95\% cuando es realizada por expertos ${ }^{(12,29)}$. En nuestro estudio encontramos que del total de CPRE realizadas, el 69,7 \% fueron exitosas. Esto debido quizá

Tabla 4. Factores propios del paciente asociados a complicaciones en la colangiopancreatografía retrógrada endoscópica.

\begin{tabular}{|c|c|c|c|c|c|c|c|}
\hline Factores & $\begin{array}{c}\text { Total } \\
(n=294)\end{array}$ & $\begin{array}{c}\text { Complicaciones } \\
(n=33)\end{array}$ & $\begin{array}{l}\text { Proporción del factor } \\
\text { en procedimiento con } \\
\text { complicaciones }(\%)\end{array}$ & $\begin{array}{l}\text { Proporción del factor } \\
\text { en procedimiento sin } \\
\text { complicaciones }(\%)\end{array}$ & OR & IC al $95 \%$ & p \\
\hline Edad > 60 años & 138 & 17 & \multirow{2}{*}{51,5} & \multirow{2}{*}{46,4} & \multirow{2}{*}{1,23} & \multirow{2}{*}{$0,55-2,72$} & \multirow{2}{*}{$0,58^{*}$} \\
\hline Edad $<=60$ años & 156 & 16 & & & & & \\
\hline Sexo femenino & 155 & 18 & \multirow{2}{*}{54,6} & \multirow{2}{*}{52,5} & \multirow{2}{*}{1,08} & \multirow{2}{*}{$0,49-2,42$} & \multirow{2}{*}{$0,86^{*}$} \\
\hline Sexo masculino & 139 & 15 & & & & & \\
\hline Con diabetes Mellitus & 26 & 4 & \multirow{2}{*}{12,1} & \multirow{2}{*}{8,43} & \multirow{2}{*}{1,49} & \multirow{2}{*}{$0,35-4,85$} & \multirow{2}{*}{$0,52^{*}$} \\
\hline Sin diabetes Mellitus & 268 & 29 & & & & & \\
\hline Con colecistectomía & 119 & 15 & \multirow{2}{*}{45,4} & \multirow{2}{*}{39,8} & \multirow{2}{*}{1,26} & \multirow{2}{*}{$0,56-2,77$} & \multirow{2}{*}{$0,57^{*}$} \\
\hline Sin colecistectomía & 175 & 18 & & & & & \\
\hline Con arritmia & 5 & 1 & \multirow{2}{*}{3,0} & \multirow{2}{*}{1,5} & \multirow{2}{*}{2,01} & \multirow{2}{*}{$0,03-21,05$} & \multirow{2}{*}{$0,45^{*}$} \\
\hline Sin arritmia & 289 & 32 & & & & & \\
\hline
\end{tabular}

* Test exacto de Fisher. N: Número de procedimientos realizados, n: Número de complicaciones. 
a que el número de esfinterotomías endoscópicas en nuestro estudio supera el $60 \%$ del total de CPRE y fueron en su mayoría de carácter terapéutico; además, un aspecto importante es el de la formación de médicos residentes, ya es indudable que las complicaciones del tutor se verán afectadas por las sucesivas curvas de aprendizaje de sus residentes, especialmente al principio de su formación ${ }^{(30)}$.

En la literatura mundial se describe que las complicaciones ocurren en aproximadamente el $10 \%$ de todos los procedimientos y la mortalidad es de alrededor del $1 \%$ $(18,31)$. Estudios retrospectivos y prospectivos reportan complicaciones con porcentajes que varían entre $2 \%$, hasta $11 \%{ }^{(2,32-36)}$. Un estudio realizado en nuestro medio por Gómez et al., reportó un 5,9\% de complicaciones ${ }^{(37)}$; otro realizado por Yriberry et al., describen sólo 3,98\% de complicaciones; no obstante, también reporta un caso de defunción post-CPRE ${ }^{38)}$. Zamalloa et al., informaron un $59,33 \%$ de éxito, muy por debajo del promedio mundial ${ }^{(39)}$, por su parte Morán et al., dieron a conocer un éxito técnico completo de $54 \%$ y un éxito clínico en el $89 \%{ }^{(40)}$, distinción que no fue hecha en el presente estudio. Finalmente, Vandervoort et al, encontraron una proporción de complicaciones de 11,2\%. En este estudio se encontró un $11,2 \%$ de complicaciones post CPRE, siendo los resultados comparables al promedio mundial de complicaciones contempladas para este procedimiento, sin ningún caso de muerte registrado.

La gran mayoría de los estudios definen las complicaciones de la CPRE según el Consenso de $1991^{(18)}$. Según estos criterios, y, por ejemplo, con respecto a la hemorragia postesfinterotomía, comienza a considerarse complicación leve al descenso de la hemoglobina superior a $2 \mathrm{gr} / \mathrm{dl}$. El sangrado endoscópico durante el procedimiento, si no hay descenso de las cifras hemáticas, no se considera como complicación. Sin embargo, la utilización de medidas hemostásicas para detener una hemorragia, puede aumentar enormemente la complejidad de la CPRE, aunque no quede reflejada como complicación. Con respecto a la pancreatitis aguda, la definición habitual es la elevación de la amilasa, al menos tres veces, por encima del valor normal, dentro de las primeras 24 horas post-CPRE, junto con dolor abdominal, que requiere estancia hospitalaria ${ }^{(41)}$.

La prevalencia de pancreatitis post-CPRE, varía entre 1,8 a $7,2 \%{ }^{(42)}$. Freeman et al., reportaron una prevalencia de complicaciones post-CPRE de 9,8\% y pancreatitis en $5,4 \%$. Por el contrario, el estudio de Loperfido et al., reportó sólo una prevalencia del $4 \%$ de complicaciones y pancreatitis post-CPRE en el 1,3\%. Sin embargo, el estudio de Freeman sólo consideró procedimientos terapéuticos, en cambio el estudio realizado por Loperfido incluyó procedimientos diagnósticos, los cuales representan un tercio del total de procedimiento descritos (942/3316). Freeman y Loperfido señalan a la pancreatitis aguda como la complicación más frecuente, seguida de la hemorragia - 2 y $0,76 \%$, respectivamente - con una mortalidad del 0,4\% en ambas publicaciones $(19,20)$. Gómez et al. encontró pancreatitis aguda en un $3,4 \%$ y Hemorragia en $1 \%{ }^{(37)}$. En la presente investigación encontró pancreatitis aguda como la complicación más frecuente en un $5,4 \%$ seguida de Hemorragia en un $4,4 \%$, no hallándose ningún caso de perforación de la vía biliar, y como ya se ha remarcado, ni de muerte. Estos valores se encuentran dentro del promedio reportado en la literatura mundial.

Con el inicio de la CPRE con esfinterotomía y precorte de la papila, se pensó en la probabilidad de la estenosis de esta y por tanto, en la posibilidad de colangitis crónica y formación de novo de cálculos: sin embargo, Masci et al. en un estudio prospectivo no evidenciaron clínicamente ninguna de estas complicaciones ${ }^{(16)}$. En este estudio tampoco se encontró ninguna asociación significativa entre la esfinterotomía y precorte con la aparición de colangitis $\mathrm{u}$ otras complicaciones.

Freeman et al., reportaron un incremento del riesgo de pancreatitis en pacientes que van a una esfinterotomía de precorte ${ }^{(14)}$. Se considera el precorte en casos extremos de canulación difícil; en nuestro estudio fue usado en el 3,7\%, no asociándose con la aparición de complicaciones.

Se ha sugerido que la edad avanzada constituye un incremento en el riesgo de pancreatitis post-CPRE ${ }^{(43)}$, esto no se pudo ver en el presente estudio como un factor estadísticamente significativo, probablemente debido a que el procedimiento (CPRE) realizado a estos pacientes, son en su mayoría practicados por el tutor altamente especializado.

La canulación repetida del conducto pancreático ha demostrado aumentar el riesgo de padecer complicaciones post-CPRE como pancreatitis aguda. En nuestro estudio, la canulación del pancreático en más de una oportunidad, aumentó el riesgo de complicaciones, como se describe en estudios previos ${ }^{(2)}$.

Se menciona que el tratamiento de elección de la coledocolitiasis es la esfinterotomía endoscópica y la extracción del cálculo ${ }^{(44)}$, siendo esto confirmado en nuestro estudio, aunque en el $21 \%$ de los casos se puede presentar la migración espontánea del cálculo ${ }^{(45)}$.

La CPRE es un procedimiento complejo que no pueden realizar todos los endoscopistas ${ }^{(29)}$. Es necesaria, no 
sólo una larga curva de aprendizaje, sino, también, un mantenimiento de la destreza adquirida, por medio de la realización constante y habitual de exploraciones. Lo ideal sería que menos endoscopistas realizaran más CPRE (31); sin embargo, no se puede evitar la formación de médicos residentes para la realización de este procedimiento. Todo esto es entendido en nuestro hospital donde la formación de residentes esta a cargo de dos médicos gastroenterólogos endoscopistas altamente entrenados.

El porcentaje de complicaciones post-CPRE, así como de éxito en este procedimiento que muestra nuestro estudio en el Hospital Nacional Guillermo Almenara Irigoyen, se encuentra dentro del promedio reportado en muchos centros a nivel mundial donde se realiza este procedimiento.

Es importante tomar en consideración que al ser este estudio una serie retrospectiva, trae consigo limitaciones propias de ese tipo de estudios, y que no ha permitido, por ejemplo, tener mediciones de marcadores biológicos importantes en la totalidad de los pacientes, que complementarían la información presentada. Asimismo, al no haber efectuado un cálculo del tamaño muestral considerando la potencia estadística puede haber influido que no se hayan encontrado diferencias significativas entre las proporciones de exposición en los pacientes con complicación y sin complicación.

Se recomienda continuar con futuros estudios donde se observe la variación de los marcadores bioquímicos post CPRE en función al tiempo; asimismo, marcadores de predicción de riesgo de complicación. De otro lado, se sugiere continuar con estudios prospectivos donde se mida el real impacto de los médicos en entrenamiento (médicos residentes en gastroenterología) en la presentación de complicaciones.

\section{Fuente de Financiamiento}

Autofinanciado.

\section{Conflictos de Interés}

Los autores manifiestan no tener conflictos de interés en la publicación de este artículo.

\section{REFERENCIAS BIBLIOGRÁFICAS}

1. Kawai K, Akasaka Y, Murakami K, Tada M, Koli Y. Endoscopic sphincterotomy of the ampulla of Vater. Gastrointest Endosc. 1974; 20(4): 148-51.

2. Vandervoort J, Soetikno RM, Tham TC, Wong RC, Ferrari AP, Montes $\mathrm{H}$, et al. Risk factors for complications after performance of ERCP. Gastrointest Endosc. 2002; 56(5): 652-56.
3. Adler DG, Baron TH, Davila RE, Egan J, Hirota WK, Leighton JA, et al. ASGE guidelines for clinical application. The role of ERCP in diseases of the biliary tract and pancreas. American Society for Gastrointestinal Endoscopy. Gastrointest Endosc. 1999; 50(6): 915-20.

4. Vennes JA, Ament M, Boyce HW Jr, Cotton PB, Jensen DM, Ravich WJ, et al. Principles of training in gastrointestinal endoscopy. American Society for Gastrointestinal Endoscopy. Standards of Training Committees. 1989-1990. Gastrointest Endosc. 1992; 38(6): 743-46.

5. Cotton PB, Williams CB. Practical gastrointestinal endoscopy. 2th. ed. Oxford: Blackwell Scientific; 1992.

6. Brizuela QR, Ruiz TJ, Fábregas RC, Martínez LR, Pernia GL, Díaz-Canel FO. Aplicaciones diagnósticas y terapéuticas de la colangiopancreatografía retrógrada endoscópica. Rev Cubana Med Milit. 2000; 29(3): 162-67.

7. Strasberg SM. Laparoscopic biliary surgery. Gastroenterol Clin North Am. 1999; 28(1): 117-32.

8. [No authors listed]. Antibiotic prophylaxis for gastrointestinal endoscopy. American Society for Gastrointestinal Endoscopy. Gastrointest Endosc. 1995; 42(6): 630-35.

9. Hirota WK, Petersen K, Baron TH, Goldstein JL, Jacobson BC, Leighton JA, et al. Guidelines for antibiotic prophylaxis for GI endoscopy. Gastrointest Endosc. 2003; 58(4): 475-82.

10. Ciocirlan M, Ponchon T. Diagnostic endoscopic retrograde cholangiopancreatography. Endoscopy. 2004; 36(2): $137-$ 46.

11. Feussner H. Endoscopic papillotomy: surgery should not be forgotten. Endoscopy. 1998; 30(9): 228-30.

12. Huibregtse K, Kimmey MB. Endoscopic retrograde cholangiopancreatography, endoscopic sphincterotomy and stone removal, and endoscopic biliary and pancreatic drainage. In: Yamada T, ed. Textbook of gastroenterology. Philadelphia: J.B. Lippincott; 1995.

13. Costamagna G, Bianco MA, Rotondano G. Costeffectiveness of endoscopic sphincterotomy. Endoscopy. 1998; 30(9): 212-15.

14. Freeman ML, DiSario JA, Nelson DB, Fennerty MB, Lee JG, Biorkman DJ, et al. Risk factors for post-ERCP pancreatitis: a prospective, multicenter study. Gastrointest Endosc. 2001; 54(4): 425-34.

15. Cheng CL, Sherman S, Watkins JL, Barnett J, Freeman M, Geenen J, et al. Risk factors for post-ERCP pancreatitis: a prospective multicenter study. The Am J Gastroenterol. 2006; 101(1): 139-47.

16. Masci E, Toti G, Mariani A, Curioni S, Lomazzi A, Dinelli M, et al. Complications of diagnostic and therapeutic ERCP: a prospective multicenter study. Am J Gastroenterol. 2001; 96(2): 417-23.

17. Christoforidis E, Goulimaris I, Kanellos I, Tsalis K, Demetriades C, Betsis D. Post-ERCP pancreatitis and hyperamylasemia: patient-related and operative risk factors. Endoscopy. 2002; 34(4): 286-92.

18. Cotton PB, Lehman G, Vennes J, Geenen JE, Russell RC, Meyers WC, et al. Endoscopic sphincterotomy complications and their management: an attempt at consensus. Gastrointest Endosc. 1991; 37(3): 383-93. 
19. Loperfido S, Angelini G, Benedetti G, Chilovi F, Costan F, De Berardinis F, et al. Major early complications from diagnostic and therapeutic ERCP: a prospective multicenter study. Gastrointest Endosc. 1998; 48(1): 1-10.

20. Freeman ML, Nelson DB, Sherman S, Haber GB, Herman ME, Dorsher PJ, et al. Complications of endoscopic biliary sphincterotomy. N Engl J Med. 1996; 335(13): 909-18.

21. Kounis NG, Zavras GM, Papadaki PJ, Kouni SN, Batsolaki M, Gouvelou-Deligianni GV, et al. Electrocardiographic changes in elderly patients during endoscopic retrograde cholangiopancreatography. Can J Gastroenterol. 2003; 17(9): 539-44.

22. Christensen M, Hendel HW, Rasmussen V, Hojgaard L, Schulze S, Rosenberg J. Endoscopic retrograde cholangiopancreatography causes reduced myocardial blood flow. Endoscopy. 2002; 34(10): 797-800.

23. Gangi S, Saidi F, Patel K, Johnstone B, Jaeger J, Shine D. Cardiovascular complications after $\mathrm{Gl}$ endoscopy: occurrence and risks in a large hospital system. Gastrointest Endosc. 2004; 60(5): 679-85.

24. Seinela L, Reinikainen P, Ahvenainen J. Effect of upper gastrointestinal endoscopy on cardiopulmonary changes in very old patients. Arch Gerontol Geriatr. 2003; 37(1): 2532

25. Fisher L, Fisher A, Thomson A. Cardiopulmonary complications of ERCP in older patients. Gastrointest Endosc. 2006; 63(7): 948-55.

26. Rabenstein T, Schneider HT, Nicklas M, Ruppert T, Katalinic A, Hahn EG, et al. Impact of skill and experience of the endoscopist on the outcome of endoscopic sphincterotomy techniques. Gastrointest Endosc. 1999; 50(5): 628-36.

27. Cotton PB, Garrow DA, Gallagher J, Romagnuolo J. Risk factors for complications after ERCP: a multivariate analysis of 11,497 procedures over 12 years. Gastrointest Endosc. 2009; 70(1): 80-88.

28. Mallery JS, Baron TH, Dominitz JA, Goldstein JL, Hirota WK, Jacobson BC, et al. Complications of ERCP. Gastrointest Endosc. 2003; 57(6): 633-38.

29. Baillie J. ERCP training: for the few, not for all. Gut. 1999; 45(1): 9-10.

30. Rodriguez Munoz S. Towards safer ERCP: selection, experience and prophylaxis. Rev Esp Enferm Dig. 2004; 96(3): 155-62.

31. Huibregtse K. Complications of endoscopic sphincterotomy and their prevention. N Engl J Med. 1996; 335(13): 96163.

32. Soehendra N. [Technique, difficulties and results of endoscopic retrograde cholangio-pancreatography (ERCP)]. Chirurg. 1977; 48(2): 98-104.

33. Leese T, Neoptolemos JP, Carr-Locke DL. Successes, failures, early complications and their management following endoscopic sphincterotomy: results in 394 consecutive patients from a single centre. Br J Surg. 1985; 72(3): 215-19.
34. Vaira D, D'Anna L, Ainley C, Dowsett J, Williams S, Baillie J, et al. Endoscopic sphincterotomy in 1000 consecutive patients. Lancet. 1989; 2(8660): 431-44.

35. Wang $P$, Li ZS, Liu F, Ren $X$, Lu NH, Fan ZN et al. Risk factors for ERCP-related complications: a prospective multicenter study. Am J Gastroenterol. 2009; 104(1): 31 40.

36. Peñaloza-Ramírez A, Leal-Buitrago C, RodríguezHernández A. Adverse events of ERCP at San José Hospital of Bogotá (Colombia). Rev Esp Enferm Dig. 2009; 101(12): 837-49.

37. Gomez Ponce RL. Complicaciones tempranas de colangiopancreatografía retrógrada endoscópica de enero de 1998 a diciembre del 2000 en el Hospital Nacional Daniel Alcides Carrión Lima-Perú. Rev Gastroenterol Peru. 2002; 22(1): 33-43.

38. Yriberry US, Salazar MF, Monge ZV, Prochazka ZR, Vila GS, Barriga BJ, et al. Eventos adversos esperados e inesperados en la endoscopía terapéutica de la vía biliar(CPRE): Experiencia en un centro privado nacional con 1356 casos consecutivos (1999-2008). Rev Gastroenterol Peru. 2009; 29(4): 311-20

39. Zamalloa H, Valdivia RM, Vargas CG, Astete BM, Núñez CN, Chávez RM, et al. Experiencia con la colangiografía retrógrada endoscópica como procedimiento diagnóstico y terapéutico. Rev Soc Peru Med Interna. 2006; 19(2): 3746.

40. Moran TL, Cumpa QR, Vargas CG, Astete BM, Valdivia RM. Características de la colangiopancreatografía retrógrada endoscópica en un centro de referencia nacional Rev Gastroenterol Perú. 2005; 25(2): 161-67.

41. Garcia-Cano LJ, Gonzalez MJ, Morillas AJ, Perez SA. Complications of endoscopic retrograde cholangiopancreatography. A study in a small ERCP unit. Rev Esp Enferm Dig. 2004; 96(3): 163-73.

42. Barr LL, Frame BC, Coulanjon A. Proposed criteria for preoperative endoscopic retrograde cholangiography in candidates for laparoscopic cholecystectomy. Surg Endosc. 1999; 13(8): 778-81.

43. Mee AS, Vallon AG, Croker JR, Cotton PB. Nonoperative removal of bile duct stones by duodenoscopic sphincterotomy in the elderly. Br Med J (Clin Res Ed). 1981 283(6290): 521-23.

44. Silvis SE. Current status of endoscopic sphincterotomy. Am J Gastroenterol. 1984; 79(9):731-3.

45. Lambert ME, Betts CD, Hill J, Faragher EB, Martin DF, Tweedle DE. Endoscopic sphincterotomy: the whole truth Br J Surg. 1991; 78(4): 473-76.

Correspondencia: Ángel Quispe-Mauricio.

Dirección: 60 Rue d'Aubervilliers, Paris, Francia.

Correo electrónico: angelquispe2@gmail.com 